\title{
Three-Dimensional Integrated Guidance and Control for Near Space Interceptor Based on Robust Adaptive Backstepping Approach
}

\author{
Changsheng Gao, ${ }^{1}$ Chunwang Jiang, ${ }^{1}$ Yan Zhang, ${ }^{2}$ and Wuxing Jing ${ }^{1}$ \\ ${ }^{1}$ School of Astronautics, Harbin Institute of Technology, Harbin 150001, China \\ ${ }^{2}$ Shenyang Aircraft Design \& Research Institute, Shenyang 110035, China \\ Correspondence should be addressed to Chunwang Jiang; cwjiang2010@163.com
}

Received 17 April 2016; Revised 5 October 2016; Accepted 12 October 2016

Academic Editor: Kenneth M. Sobel

Copyright (c) 2016 Changsheng Gao et al. This is an open access article distributed under the Creative Commons Attribution License, which permits unrestricted use, distribution, and reproduction in any medium, provided the original work is properly cited.

\begin{abstract}
This study presents a novel integrated guidance and control method for near space interceptor, considering the coupling among different channels of the missile dynamics, which makes the most of the overall performance of guidance and control system. Initially, three-dimensional integrated guidance and control model is employed by combining the interceptor-target relative motion model with the nonlinear dynamics of the interceptor, which establishes a direct relationship between the interceptor-target relative motion and the deflections of aerodynamic fins. Subsequently, regarding the acceleration of the target as bounded uncertainty of the system, an integrated guidance and control algorithm is designed based on robust adaptive backstepping method, with the upper bound of the uncertainties unknown. Moreover, a nonlinear tracking differentiator is introduced to reduce the "compute explosion" caused by backstepping method. It is proved that tracking errors of the state and the upper bound of the uncertainties converge to the neighborhoods of the origin exponentially. Finally, simulations results show that, compared to the conventional guidance and control design, the algorithm proposed in this paper has greater advantages in miss distance, required normal overload, and flight stability, especially when attacking high-maneuvering targets.
\end{abstract}

\section{Introduction}

The guidance and the control systems of interceptors are complicated because they are nonlinear and coupled with each other. On the one hand, the guidance system guides the interceptor to the target, during which the variation of trajectory parameters intensifies the uncertainty of the attitude model and brings a burden to the control of attitude. On the other hand, the control system adjusts the attitude in order to track the guidance command, during which the action of the actuators exerts influence on the trajectory and the autopilot lag reduces the performance of the guidance system. Conventionally, guidance and control systems are designed separately, which is proved efficient and convenient merely for the interception of nonmaneuvering target. However, the conventional design approach does not make full use of the coupling between guidance and control and hence has great limitations in improving the overall performance of the interceptor. For the near space interceptor, especially when attacking high-maneuvering target, the coupling between guidance and control is significant while the conventional design method may result in deterioration of the overall performance, sometimes even failure of the interception. Therefore, the study of integrated guidance and control, which makes full use of the coupling between the guidance and the control, is of great significance for better overall performance of the interceptor and higher accuracy for near space interception.

Integrated guidance and control design can be mainly classified into two categories: the partial one and the complete one. Partial integrated guidance and control is that the guidance law is designed accounting for autopilot dynamics, by which the negative influence of autopilot lag is compensated. In [1-3], guidance laws are studied for the near space interception of maneuvering target, in which the autopilot is approximated as first-order dynamics. Actually, the autopilot 
is of high-order dynamics. As an improvement, guidance laws in [4-6] are designed accounting for the autopilot as secondorder dynamics. In [7-9], finite-time convergence guidance laws in the presence of autopilot lag are studied, which guarantee that the line-of-sight angular rate converges to zero in finite time. For better attack performance, guidance laws with impact angle constraints and autopilot dynamics compensation are designed in [10-12]. Compared to conventional design approach, partial integrated guidance and control can improve the overall performance of the system to some extent, in which the coupling between guidance and control is approximately considered. However, partial integrated guidance and control still keeps the outline of conventional design scheme, that is, the outer guidance loop and inner control loop, which has some limitations in improving the overall performance. On the one hand, the coupling between guidance and control is normally approximated as the firstorder dynamics or the second-order one, both of which cannot represent the actual coupling. On the other hand, all the couplings are regarded as disturbances to compensate, resulting in the underutilization of conductive couplings.

Complete integrated guidance and control is that the guidance and control is designed as a whole and the control command is produced directly according to the interceptortarget relative motion, which breaks through the outline of conventional design scheme entirely. Complete integrated guidance and control has great advantage in making full use of the coupling between guidance and control and improving the overall performance of the system, which is the trend of the guidance and control design. For convenience, most of the complete integrated guidance and control are studied in two-dimensional space [13-19]. Actually, all the near space interceptions are conducted in three-dimensional space, so achievements of two-dimensional integrated guidance and control cannot be applied in three-dimensional interception directly. By viewing the coupling among the pitch, yaw, and roll channels as external disturbances, three-channel independent integrated guidance and control are studied in [20-23], assumption of which is that the movement of yaw and roll channels is in a small range. When attacking high-maneuvering target, however, this assumption cannot be satisfied, which limits the application of three-channel independent integrated guidance and control. To realize three-channel coupling integrated guidance and control for interception of maneuvering target, three-dimensional integrated guidance and control model of strict feedback cascade is established in [24-26], based on which integrated guidance and control algorithms are designed using dynamic surface control. Meanwhile, robust functions $[24,25]$ or extended state observer [26] are introduced to compensate for the disturbances. In [27], three-dimensional full states coupling integrated guidance and control model is established and $L_{1}$ adaptive integrated guidance and control algorithm is designed. But the resulting control algorithm is relatively complicated and hard to be realized in engineering.

Based on the idea of complete integrated guidance and control, a novel integrated guidance and control considering the coupling among three channels is proposed in this paper, aiming at three-dimensional near space interception

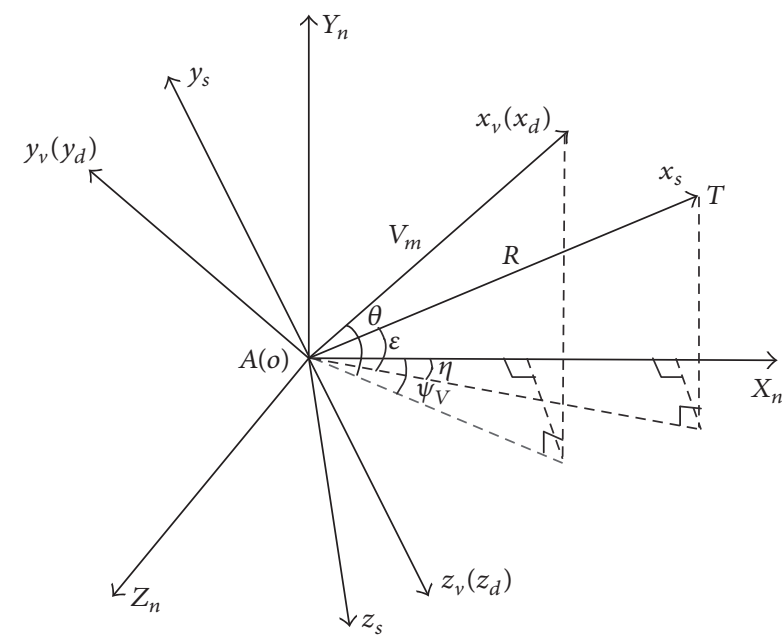

FIGURE 1: Three-dimensional interceptor-target engagement geometry.

of maneuvering target. Firstly, considering the coupling among the pitch, yaw, and roll channels, three-dimensional integrated guidance and control model is built, which establishes the direct relationship between the interceptortarget relative motion and the deflections of aerodynamic fins. Secondly, regarding the acceleration of the target as bounded uncertainty of the system, an integrated guidance and control algorithm is designed based on robust adaptive backstepping method, with no information of the upper bound of the uncertainties. Thirdly, a nonlinear tracking differentiator is introduced to reduce the "compute explosion" caused by backstepping method. It is proved that tracking errors of the state and the unknown parameters converge to the neighborhoods of the origin exponentially. Finally, simulation and comparison are conducted between the proposed integrated guidance and control method and the conventional guidance and control design. Simulation results show that the proposed algorithm in this paper has greater advantages in miss distance, required normal overload, and flight stability, especially when attacking the highmaneuvering target.

\section{Integrated Guidance and Control Model}

The interceptor-target engagement geometry in threedimensional space is shown in Figure 1, where $A X_{n} Y_{n} Z_{n}$ and $o x_{s} y_{s} z_{s}$ are, respectively, the inertial and line-of-sight coordinate systems; $o x_{v} y_{v} z_{v}$ and $o x_{d} y_{d} z_{d}$ are, respectively, the velocity and half velocity coordinate systems; $o$ and $T$ represent the centroid of the interceptor and target, respectively. Supposing that skid-to-turn (STT) control scheme is adopted in the flight of interceptor, the velocity coordinate system coincides with half velocity coordinate system.

According to the above definitions, integrated guidance and control model for near space interceptor in three- 
dimensional space can be described as the following form [24]:

$$
\begin{aligned}
& \dot{\mathbf{x}}_{0}=\mathbf{f}_{0}\left(\mathbf{x}_{0}\right)+\mathbf{g}_{0}\left(\mathbf{x}_{0}\right) \mathbf{x}_{1}^{*}+\Delta_{0}, \\
& \dot{\mathbf{x}}_{1}=\mathbf{f}_{1}\left(\mathbf{x}_{1}\right)+\mathbf{g}_{1}\left(\mathbf{x}_{1}\right) \mathbf{x}_{2}+\Delta_{1}, \\
& \dot{\mathbf{x}}_{2}=\mathbf{f}_{2}\left(\mathbf{x}_{1}, \mathbf{x}_{2}\right)+\mathbf{g}_{2} \mathbf{u}+\Delta_{2}, \\
& \mathbf{y}=\mathbf{x}_{0},
\end{aligned}
$$

where the state vectors are defined as $\mathbf{x}_{0}=\left[\begin{array}{cc}\dot{\varepsilon} & \dot{\eta}\end{array}\right]^{T}, \mathbf{x}_{1}=$ $\left[\begin{array}{ll}\mathbf{x}_{1}^{*} & \gamma\end{array}\right]^{T}=\left[\begin{array}{lll}\alpha & \beta & \gamma\end{array}\right]^{T}$, and $\mathbf{x}_{2}=\left[\begin{array}{lll}\omega_{x} & \omega_{y} & \omega_{z}\end{array}\right]^{T}$; the input vector is defined as $\mathbf{u}=\left[\begin{array}{lll}\delta_{x} & \delta_{y} & \delta_{z}\end{array}\right]^{T}$; the output vector is defined as $\mathbf{y}=\left[\begin{array}{ll}\dot{\varepsilon} & \dot{\eta}\end{array}\right]^{T} ; \Delta_{i}, i=0,1,2$, are the bounded uncertainties of the system, including the modeling errors and external disturbances; concrete expressions of $\mathbf{f}_{0}\left(\mathbf{x}_{0}\right)$, $\mathbf{g}_{0}\left(\mathbf{x}_{0}\right), \mathbf{f}_{1}\left(\mathbf{x}_{1}\right), \mathbf{g}_{1}\left(\mathbf{x}_{1}\right), \mathbf{f}_{2}\left(\mathbf{x}_{1}, \mathbf{x}_{2}\right)$, and $\mathbf{g}_{2}$ are as follows:

$$
\begin{aligned}
& \mathbf{f}_{0}\left(\mathbf{x}_{0}\right)=\left[\begin{array}{c}
-\frac{2 \dot{R} \dot{\varepsilon}+R \dot{\eta}^{2} \sin \varepsilon \cos \varepsilon}{R} \\
\frac{-2 \dot{R} \dot{\eta} \cos \varepsilon+2 R \dot{\varepsilon} \dot{\eta} \sin \varepsilon}{R \cos \varepsilon}
\end{array}\right] \text {, } \\
& \mathbf{g}_{0}\left(\mathbf{x}_{0}\right)=\left[\begin{array}{cc}
-\frac{a_{22} q S C_{y}^{\alpha}}{m R} & -\frac{a_{23} q S C_{z}^{\beta}}{m R} \\
\frac{a_{32} q S C_{y}^{\alpha}}{m R \cos \varepsilon} & \frac{a_{33} q S C_{z}^{\beta}}{m R \cos \varepsilon}
\end{array}\right], \\
& \mathbf{f}_{1}\left(\mathbf{x}_{1}\right)=\left[\begin{array}{c}
\frac{-q S C_{y}^{\alpha} \alpha}{(m V \cos \beta)} \\
\frac{q S C_{z}^{\beta} \beta}{(m V)} \\
0
\end{array}\right] \\
& \mathbf{g}_{1}\left(\mathbf{x}_{1}\right)=\left[\begin{array}{ccc}
-\tan \beta \cos \alpha & \tan \beta \sin \alpha & 1 \\
\sin \alpha & \cos \alpha & 0 \\
1 & -\tan \vartheta \cos \gamma & \tan \vartheta \sin \gamma
\end{array}\right], \\
& \mathbf{f}_{2}\left(\mathbf{x}_{1}, \mathbf{x}_{2}\right) \\
& =\left[\begin{array}{c}
\frac{\left[q S L\left(m_{x}^{\bar{\omega}_{x}} \omega_{x} L / V\right)+\left(I_{y}-I_{z}\right) \omega_{y} \omega_{z}\right]}{I_{x}} \\
\frac{\left[q S L\left(m_{y}^{\beta} \beta+m_{y}^{\bar{\omega}_{y}} \omega_{y} L / V\right)+\left(I_{z}-I_{x}\right) \omega_{z} \omega_{x}\right]}{I_{y}} \\
{\left[q S L\left(m_{z}^{\alpha} \alpha+m_{z}^{\bar{\omega}_{z}} \omega_{z} L / V\right)+\left(I_{x}-I_{y}\right) \omega_{x} \omega_{y}\right]} \\
I_{z}
\end{array}\right], \\
& \mathbf{g}_{2}=\operatorname{diag}\left(\left[\begin{array}{lll}
\frac{q S L m_{x}^{\delta_{x}}}{I_{x}} & \frac{q S L m_{y}^{\delta_{y}}}{I_{y}} & \frac{q S L m_{z}^{\delta_{z}}}{I_{z}}
\end{array}\right]\right), \\
& a_{22} \\
& =\sin \theta \cos \psi_{V} \sin \varepsilon \cos \eta+\cos \theta \cos \varepsilon \\
& +\sin \theta \sin \psi_{V} \sin \varepsilon \sin \eta,
\end{aligned}
$$

$$
\begin{aligned}
& a_{23}=-\sin \psi_{V} \sin \varepsilon \cos \eta+\cos \psi_{V} \sin \varepsilon \sin \eta \\
& a_{32}=-\sin \theta \cos \psi_{V} \sin \eta+\sin \theta \sin \psi_{V} \cos \eta \\
& a_{33}=\sin \psi_{V} \sin \eta+\cos \psi_{V} \cos \eta .
\end{aligned}
$$

From the integrated guidance and control model of (1), we can see that the direct relationship between the interceptortarget relative motion and the deflections of aerodynamic fins is built, in which the coupling among the pitch, yaw, and roll channels is considered sufficiently. What is more, the state variables of (1) are of cascaded feedback form. That is,

$$
\left.\left[\begin{array}{c}
\dot{\varepsilon} \\
\dot{\eta}
\end{array}\right]\right)\left[\begin{array}{c}
\alpha \\
\beta \\
\gamma
\end{array}\right] \longrightarrow\left[\begin{array}{c}
\omega_{x} \\
\omega_{y} \\
\omega_{z}
\end{array}\right] \longrightarrow\left[\begin{array}{c}
\delta_{x} \\
\delta_{y} \\
\delta_{z}
\end{array}\right]
$$

\section{Integrated Guidance and Control Algorithm}

3.1. Robust Adaptive Backstepping Controller. Before the controller design process, some assumptions are made as follows.

Assumption 1. $\mathbf{g}_{i}, i=0,1,2$, are bounded and invertible.

Assumption 2. There exist a set of unknown positive constant $\rho_{i}$ and known nonnegative smooth function $\delta_{i}(x, t)$, such that $\left\|\Delta_{i}\right\| \leq \rho_{i} \delta_{i}(x, t), \quad i=0,1,2$.

Design objective of integrated guidance and control is selected to be zero line-of-sight angular rate. What is more, roll angle of STT interceptor should be kept zero during the whole flight. That is,

$$
\mathbf{x}_{0} \longrightarrow \mathbf{x}_{0 d}=\left[\begin{array}{ll}
0 & 0
\end{array}\right]^{T} ; \quad \gamma=0 .
$$

Based on robust adaptive backstepping approach, integrated guidance and control algorithm can be designed as follows.

Step 1. The tracking error of state $\mathbf{x}_{0}$ is defined as

$$
\mathbf{z}_{0}=\mathbf{x}_{0}-\mathbf{x}_{0 d}
$$

and the derivative of $\mathbf{z}_{0}$ with respect to time is given by

$$
\dot{\mathbf{z}}_{0}=\dot{\mathbf{x}}_{0}-\dot{\mathbf{x}}_{0 d}=\mathbf{f}_{0}\left(\mathbf{x}_{0}\right)+\mathbf{g}_{0}\left(\mathbf{x}_{0}\right) \mathbf{x}_{1}^{*}+\Delta_{0}-\dot{\mathbf{x}}_{0 d} .
$$

According to exponential approaching law, the virtual control $\mathbf{x}_{1 d}^{*}$ is constructed as the following form:

$$
\mathbf{x}_{1 d}^{*}=-\mathbf{g}_{0}\left(\mathbf{x}_{0}\right)^{-1}\left[\mathbf{k}_{0} \mathbf{z}_{0}+\mathbf{f}_{0}\left(\mathbf{x}_{0}\right)+\boldsymbol{\eta}_{0}-\dot{\mathbf{x}}_{0 d}\right],
$$

where $\mathbf{k}_{0}=\operatorname{diag}\left(\left[k_{01} k_{02}\right]\right)$ is the gain matrix with symmetric positive definite form; $\boldsymbol{\eta}_{0}$ is the robust function introduced to compensate for the uncertainty $\Delta_{0}$, which is given by [28]

$$
\boldsymbol{\eta}_{0}=\mathbf{z}_{0} \widehat{\rho}_{0}^{2} \delta_{0}^{2}+\frac{\varepsilon_{0}^{2}}{4} \mathbf{z}_{0} \delta_{0}^{2}
$$

where $\varepsilon_{0}$ is the gain parameter; $\widehat{\rho}_{0}$ is the approximation of the unknown positive constant $\rho_{0}$, which can be designed by the 
following adaptive algorithm:

$$
\dot{\hat{\rho}}_{0}=r_{0}\left[\varepsilon_{0}\left\|\mathbf{z}_{0}\right\|^{2} \delta_{0}^{2}-\sigma_{0}\left(\widehat{\rho}_{0}-\rho_{0}^{0}\right)\right] \text {, }
$$

where $r_{0}, \sigma_{0}$, and $\rho_{0}^{0}$ are positive design parameters.

Step 2. Let $\mathbf{x}_{1 d}=\left[\begin{array}{ll}\mathbf{x}_{1 d}^{*} & 0\end{array}\right]^{T}$; then $\dot{\mathbf{x}}_{1 d}=\left[\begin{array}{ll}\dot{\mathbf{x}}_{1 d}^{*} & 0\end{array}\right]^{T}$. The tracking error of state $\mathbf{x}_{1}$ is defined as

$$
\mathbf{z}_{1}=\mathbf{x}_{1}-\mathbf{x}_{1 d}
$$

and the derivative of $\mathbf{z}_{1}$ with respect to time is given by

$$
\dot{\mathbf{z}}_{1}=\dot{\mathbf{x}}_{1}-\dot{\mathbf{x}}_{1 d}=\mathbf{f}_{1}\left(\mathbf{x}_{1}\right)+\mathbf{g}_{1}\left(\mathbf{x}_{1}\right) \mathbf{x}_{2}+\Delta_{1}-\dot{\mathbf{x}}_{1 d} .
$$

The virtual control $\mathbf{x}_{2 d}$ is constructed as the following form:

$$
\begin{aligned}
\mathbf{x}_{2 d} & =-\mathbf{g}_{1}\left(\mathbf{x}_{1}\right)^{-1} \\
\cdot & {\left[\mathbf{k}_{1} \mathbf{z}_{1}+\mathbf{f}_{1}\left(\mathbf{x}_{1}\right)+\boldsymbol{\eta}_{1}-\dot{\mathbf{x}}_{1 d}+\left[\begin{array}{ll}
\mathbf{z}_{0}^{T} \mathbf{g}_{0}\left(\mathbf{x}_{0}\right) & 0
\end{array}\right]^{T}\right], }
\end{aligned}
$$

where $\mathbf{k}_{1}=\operatorname{diag}\left(\left[\begin{array}{lll}k_{11} & k_{12} & k_{13}\end{array}\right]\right)$ is the gain matrix with symmetric positive definite form; $\left[\mathbf{z}_{0}^{T} \mathbf{g}_{0}\left(\mathbf{x}_{0}\right) \quad 0\right]^{T}$ is introduced to compensate for the coupling between $\mathbf{z}_{1}$ and $\mathbf{z}_{0} ; \boldsymbol{\eta}_{1}$ is the robust function compensating for the uncertainty $\Delta_{1}$, which is given by

$$
\begin{aligned}
& \boldsymbol{\eta}_{1}=\mathbf{z}_{1} \widehat{\rho}_{1}^{2} \delta_{1}^{2}+\frac{\varepsilon_{1}^{2}}{4} \mathbf{z}_{1} \delta_{1}^{2}, \\
& \dot{\hat{\rho}}_{1}=r_{1}\left[\varepsilon_{1}\left\|\mathbf{z}_{1}\right\|^{2} \delta_{1}^{2}-\sigma_{1}\left(\hat{\rho}_{1}-\rho_{1}^{0}\right)\right],
\end{aligned}
$$

where $\varepsilon_{1}, r_{1}, \sigma_{1}$, and $\rho_{1}^{0}$ are positive design parameters.

Step 3. The tracking error of state $\mathbf{x}_{2}$ is defined as

$$
\mathbf{z}_{2}=\mathbf{x}_{2}-\mathbf{x}_{2 d}
$$

and the derivative of $\mathbf{z}_{2}$ with respect to time is given by

$$
\dot{\mathbf{z}}_{2}=\dot{\mathbf{x}}_{2}-\dot{\mathbf{x}}_{2 d}=\mathbf{f}_{2}\left(\mathbf{x}_{1}, \mathbf{x}_{2}\right)+\mathbf{g}_{2} \mathbf{u}+\Delta_{2}-\dot{\mathbf{x}}_{2 d} .
$$

The real control $\mathbf{u}$ is constructed as the following form:

$$
\mathbf{u}=-\mathbf{g}_{2}^{-1}\left[\mathbf{k}_{2} \mathbf{z}_{2}+\mathbf{f}_{2}\left(\mathbf{x}_{1}, \mathbf{x}_{2}\right)+\boldsymbol{\eta}_{2}-\dot{\mathbf{x}}_{2 d}+\mathbf{g}_{1}^{T}\left(\mathbf{x}_{1}\right) \mathbf{z}_{1}\right],
$$

where $\mathbf{k}_{2}=\operatorname{diag}\left(\left[\begin{array}{lll}k_{21} & k_{22} & k_{23}\end{array}\right]\right)$ is the gain matrix with symmetric positive definite form; $\mathbf{g}_{1}^{T}\left(\mathbf{x}_{1}\right) \mathbf{z}_{1}$ is introduced to compensate for the coupling between $\mathbf{z}_{2}$ and $\mathbf{z}_{1} ; \boldsymbol{\eta}_{2}$ is the robust function compensating for the uncertainty $\Delta_{2}$, which is given by

$$
\begin{aligned}
& \boldsymbol{\eta}_{2}=\mathbf{z}_{2} \widehat{\rho}_{2}^{2} \delta_{2}^{2}+\frac{\varepsilon_{2}^{2}}{4} \mathbf{z}_{2} \delta_{2}^{2}, \\
& \dot{\hat{\rho}}_{2}=r_{2}\left[\varepsilon_{2}\left\|\mathbf{z}_{2}\right\|^{2} \delta_{2}^{2}-\sigma_{2}\left(\hat{\rho}_{2}-\rho_{2}^{0}\right)\right],
\end{aligned}
$$

where $\varepsilon_{2}, r_{2}, \sigma_{2}$, and $\rho_{2}^{0}$ are positive design parameters.

3.2. Nonlinear Tracking Differentiator. It should be emphasized that, as a common drawback of classical backstepping method, the derivative of the virtual control (e.g., $\dot{\mathbf{x}}_{1 d}$ and $\dot{\mathbf{x}}_{2 d}$ ) is computationally complex, which intensifies the "computer explosion" of backstepping method. To overcome such drawback, a nonlinear tracking differentiator is introduced, by which the derivative of the virtual control can be well approximated. The discrete form of the nonlinear tracking differentiator is as follows [29]:

$$
\begin{aligned}
\widehat{v}_{1}(k+1)= & \widehat{v}_{1}(k)+h \widehat{\nu}_{2}(k), \\
\widehat{v}_{2}(k+1)= & \widehat{v}_{2}(k)+h \\
& \cdot \operatorname{fst}\left(\widehat{\nu}_{1}(k)-v(k), \widehat{v}_{2}(k), \delta, h\right),
\end{aligned}
$$

where $\nu(k)$ is the input; $\widehat{\nu}_{1}(k)$ is the approximation of $\nu(k)$; $\widehat{v}_{2}(k)$ is the approximation of the derivative of $\nu(k) ; h$ is the sampling step; $\delta$ is the gain parameter that determines the speed of tracking; fst $(\cdot)$ is the time-optimal control synthesis function, which is given by

$$
\text { fst } \begin{aligned}
\left(x_{1}, x_{2}, \delta, h\right) & = \begin{cases}-\delta \operatorname{sign}(a), & |a|>\delta h, \\
-\frac{a}{h}, & |a| \leq \delta h,\end{cases} \\
a & = \begin{cases}x_{2}+\frac{\sqrt{\delta^{2} h^{2}+8 \delta\left|x_{1}+h x_{2}\right|}-\delta h}{2} \operatorname{sign}\left(x_{1}+h x_{2}\right), & \left|x_{1}+h x_{2}\right|>\delta h^{2}, \\
x_{2}+\frac{x_{1}+h x_{2}}{h}, & \left|x_{1}+h x_{2}\right| \leq \delta h^{2} .\end{cases}
\end{aligned}
$$

Using the nonlinear tracking differentiator of (18), we have $\widehat{\nu}_{1}(k) \rightarrow \nu(k)$ and $\widehat{\nu}_{2}(k) \rightarrow \dot{\nu}(k)$. The differentiator is not only of high accuracy in the approximation of the input and its derivative, but also capable of filtering the noise within the input signal.
To analyze the performance of this differentiator, a numerical simulation is conducted, in which the gain parameter and sampling step are set as $\delta=100$ and $h=0.02 \mathrm{~s}$. The tracking results of sinusoidal input $v=\sin (t)$ and its derivative are presented in Figure 2, from which we can see 

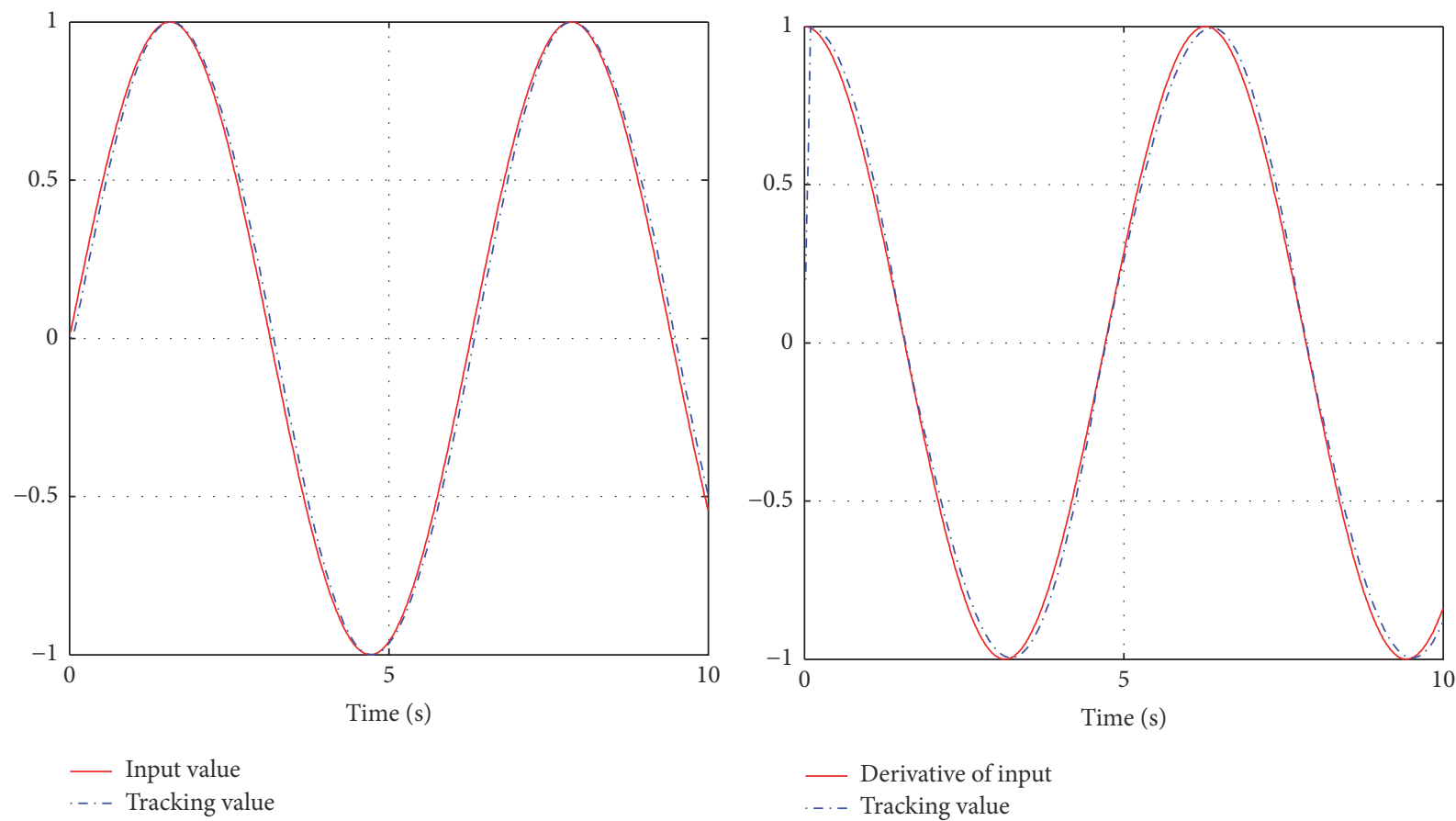

FIGURE 2: Tracking results of sinusoidal input and its derivative.

that the differentiator is of high speed and accuracy in the approximation of the input and its derivatives.

3.3. Stability Analysis. Define the candidate Lyapunov function as

$$
V=\frac{1}{2} \sum_{i=0}^{2} \mathbf{z}_{i}^{T} \mathbf{z}_{i}+\frac{1}{2} \sum_{i=0}^{2} \frac{1}{r_{i}} \widetilde{\rho}_{i}^{2}
$$

where $\tilde{\rho}_{i}$ are the tracking errors of $\rho_{i}$; that is,

$$
\tilde{\rho}_{i}=\hat{\rho}_{i}-\rho_{i}, \quad i=0,1,2 .
$$

Under Assumption 2, $\rho_{i}$ are constants; then the derivatives of $\tilde{\rho}_{i}$ satisfy

$$
\dot{\tilde{\rho}}_{i}=\dot{\hat{\rho}}_{i}, \quad i=0,1,2 .
$$

Differentiating (20) with respect to time, we have

$$
\dot{V}=\sum_{i=0}^{2} \mathbf{z}_{i}^{T} \dot{\mathbf{z}}_{i}+\sum_{i=0}^{2} \frac{1}{r_{i}} \widetilde{\rho}_{i} \dot{\tilde{\rho}}_{i}
$$

Define the tracking error of $\mathbf{x}_{1}^{*}$ as

$$
\mathbf{z}_{1}^{*}=\mathbf{x}_{1}^{*}-\mathbf{x}_{1 d}^{*} \text {. }
$$

Then, we get

$$
\begin{aligned}
\mathbf{z}_{0}^{T} \dot{\mathbf{z}}_{0} & +\frac{1}{r_{0}} \widetilde{\rho}_{0} \dot{\tilde{\rho}}_{0} \\
= & \mathbf{z}_{0}^{T}\left[\mathbf{f}_{0}\left(\mathbf{x}_{0}\right)+\mathbf{g}_{0}\left(\mathbf{x}_{0}\right)\left(\mathbf{z}_{1}^{*}+\mathbf{x}_{1 d}^{*}\right)+\Delta_{0}-\dot{\mathbf{x}}_{0 d}\right] \\
& +\frac{1}{r_{0}} \widetilde{\rho}_{0} \dot{\tilde{\rho}}_{0} .
\end{aligned}
$$

Substituting (7) and (22) into (25), we have

$$
\begin{aligned}
\mathbf{z}_{0}^{T} \dot{\mathbf{z}}_{0}+\frac{1}{r_{0}} \widetilde{\rho}_{0} \dot{\tilde{\rho}}_{0}= & -\mathbf{z}_{0}^{T} \mathbf{k}_{0} \mathbf{z}_{0}+\mathbf{z}_{0}^{T} \mathbf{g}_{0}\left(\mathbf{x}_{0}\right) \mathbf{z}_{1}^{*} \\
& +\mathbf{z}_{0}^{T}\left(\boldsymbol{\Delta}_{0}-\boldsymbol{\eta}_{0}\right)+\widetilde{\rho}_{0} \varepsilon_{0}\left\|\mathbf{z}_{0}\right\|^{2} \delta_{0}^{2} \\
& -\tilde{\rho}_{0} \sigma_{0}\left(\widehat{\rho}_{0}-\rho_{0}^{0}\right) .
\end{aligned}
$$

Since

$$
\begin{aligned}
&- \tilde{\rho}_{0} \sigma_{0}\left(\widehat{\rho}_{0}-\rho_{0}^{0}\right) \\
&=-\frac{1}{2} \sigma_{0} \tilde{\rho}_{0}^{2}-\frac{1}{2} \sigma_{0}\left(\widehat{\rho}_{0}-\rho_{0}^{0}\right)^{2}+\frac{1}{2} \sigma_{0}\left(\rho_{0}-\rho_{0}^{0}\right)^{2}, \\
& \mathbf{z}_{0}^{T}\left(\Delta_{0}-\boldsymbol{\eta}_{0}\right)+\widetilde{\rho}_{0} \varepsilon_{0}\left\|\mathbf{z}_{0}\right\|^{2} \delta_{0}^{2} \\
&=\mathbf{z}_{0}^{T}\left(\Delta_{0}-\mathbf{z}_{0} \hat{\rho}_{0}^{2} \delta_{0}^{2}-\frac{\varepsilon_{0}^{2}}{4} \mathbf{z}_{0} \delta_{0}^{2}\right)+\widetilde{\rho}_{0} \varepsilon_{0}\left\|\mathbf{z}_{0}\right\|^{2} \delta_{0}^{2} \\
& \leq\left\|\mathbf{z}_{0}\right\| \delta_{0} \rho_{0}-\left\|\mathbf{z}_{0}\right\|^{2} \hat{\rho}_{0}^{2} \delta_{0}^{2}-\frac{\varepsilon_{0}^{2}}{4}\left\|\mathbf{z}_{0}\right\|^{2} \delta_{0}^{2} \\
&+\widetilde{\rho}_{0} \varepsilon_{0}\left\|\mathbf{z}_{0}\right\|^{2} \delta_{0}^{2} \\
& \leq\left(\varepsilon_{0}\left\|\mathbf{z}_{0}\right\|^{2} \delta_{0}^{2}+\frac{1}{4 \varepsilon_{0}}\right) \rho_{0}-\left\|\mathbf{z}_{0}\right\|^{2} \widehat{\rho}_{0}^{2} \delta_{0}^{2} \\
& \quad-\frac{\varepsilon_{0}^{2}}{4}\left\|\mathbf{z}_{0}\right\|^{2} \delta_{0}^{2}+\widetilde{\rho}_{0} \varepsilon_{0}\left\|\mathbf{z}_{0}\right\|^{2} \delta_{0}^{2} \\
&=-\delta_{0}^{2}\left(\hat{\rho}_{0}\left\|\mathbf{z}_{0}\right\|-\frac{\varepsilon_{0}}{2}\left\|\mathbf{z}_{0}\right\|\right)^{2}+\frac{\rho_{0}}{4 \varepsilon_{0}} \leq \frac{\rho_{0}}{4 \varepsilon_{0}},
\end{aligned}
$$


hence, we have

$$
\begin{aligned}
\mathbf{z}_{0}^{T} \dot{\mathbf{z}}_{0}+\frac{1}{r_{0}} \widetilde{\rho}_{0} \dot{\tilde{\rho}}_{0} \leq & -\mathbf{z}_{0}^{T} \mathbf{k}_{0} \mathbf{z}_{0}+\mathbf{z}_{0}^{T} \mathbf{g}_{0}\left(\mathbf{x}_{0}\right) \mathbf{z}_{1}^{*}+\frac{\rho_{0}}{4 \varepsilon_{0}} \\
& -\frac{1}{2} \sigma_{0} \widetilde{\rho}_{0}^{2}+\frac{1}{2} \sigma_{0}\left(\rho_{0}-\rho_{0}^{0}\right)^{2} .
\end{aligned}
$$

Similarly, we get

$$
\begin{aligned}
\mathbf{z}_{1}^{T} \dot{\mathbf{z}}_{1}+\frac{1}{r_{1}} \widetilde{\rho}_{1} \dot{\tilde{\rho}}_{1} \leq & -\mathbf{z}_{1}^{T} \mathbf{k}_{1} \mathbf{z}_{1}+\mathbf{z}_{1}^{T} \mathbf{g}_{1}\left(\mathbf{x}_{1}\right) \mathbf{z}_{2} \\
& -\mathbf{z}_{1}^{T}\left[\mathbf{z}_{0}^{T} \mathbf{g}_{0}\left(\mathbf{x}_{0}\right) 0\right]^{T}+\frac{\rho_{1}}{4 \varepsilon_{1}} \\
& -\frac{1}{2} \sigma_{1} \widetilde{\rho}_{1}^{2}+\frac{1}{2} \sigma_{1}\left(\rho_{1}-\rho_{1}^{0}\right)^{2}, \\
\mathbf{z}_{2}^{T} \dot{\mathbf{z}}_{2}+\frac{1}{r_{2}} \widetilde{\rho}_{2} \dot{\tilde{\rho}}_{2} \leq & -\mathbf{z}_{2}^{T} \mathbf{k}_{2} \mathbf{z}_{2}-\mathbf{z}_{2}^{T} \mathbf{g}_{1}^{T}\left(\mathbf{x}_{1}\right) \mathbf{z}_{1}+\frac{\rho_{2}}{4 \varepsilon_{2}} \\
& -\frac{1}{2} \sigma_{2} \widetilde{\rho}_{2}^{2}+\frac{1}{2} \sigma_{2}\left(\rho_{2}-\rho_{2}^{0}\right)^{2} .
\end{aligned}
$$

It is easy to verify that

$$
\begin{aligned}
& \mathbf{z}_{0}^{T} \mathbf{g}_{0}\left(\mathbf{x}_{0}\right) \mathbf{z}_{1}^{*}=\mathbf{z}_{1}^{T}\left[\begin{array}{ll}
\mathbf{z}_{0}^{T} \mathbf{g}_{0}\left(\mathbf{x}_{0}\right) & 0
\end{array}\right]^{T}, \\
& \mathbf{z}_{1}^{T} \mathbf{g}_{1}\left(\mathbf{x}_{1}\right) \mathbf{z}_{2}=\mathbf{z}_{2}^{T} \mathbf{g}_{1}^{T}\left(\mathbf{x}_{1}\right) \mathbf{z}_{1} .
\end{aligned}
$$

Substituting (28)-(29) into (23), we have

$$
\begin{aligned}
\dot{V} \leq & -\mathbf{z}_{0}^{T} \mathbf{k}_{0} \mathbf{z}_{0}-\mathbf{z}_{1}^{T} \mathbf{k}_{1} \mathbf{z}_{1}-\mathbf{z}_{2}^{T} \mathbf{k}_{2} \mathbf{z}_{2}-\frac{1}{2} \sigma_{0} \widetilde{\rho}_{0}^{2}-\frac{1}{2} \sigma_{1} \widetilde{\rho}_{1}^{2} \\
& -\frac{1}{2} \sigma_{2} \widetilde{\rho}_{2}^{2}+\frac{\rho_{0}}{4 \varepsilon_{0}}+\frac{1}{2} \sigma_{0}\left(\rho_{0}-\rho_{0}^{0}\right)^{2}+\frac{\rho_{1}}{4 \varepsilon_{1}} \\
& +\frac{1}{2} \sigma_{1}\left(\rho_{1}-\rho_{1}^{0}\right)^{2}+\frac{\rho_{2}}{4 \varepsilon_{2}}+\frac{1}{2} \sigma_{2}\left(\rho_{2}-\rho_{2}^{0}\right)^{2} .
\end{aligned}
$$

Furthermore, we get

$$
\begin{aligned}
\dot{V} \leq & -\sum_{i=0}^{2} k_{i \min } \mathbf{z}_{i}^{T} \mathbf{z}_{i}-\sum_{i=0}^{2} \frac{1}{2} \sigma_{i} \widetilde{\rho}_{i}^{2} \\
& +\sum_{i=0}^{2}\left(\frac{\rho_{i}}{4 \varepsilon_{i}}+\frac{1}{2} \sigma_{i}\left(\rho_{i}-\rho_{i}^{0}\right)^{2}\right) \leq-k V+c,
\end{aligned}
$$

where

$$
\begin{aligned}
k & =\min \left\{\begin{array}{c}
2 k_{0 \min }, 2 k_{1 \mathrm{~min}}, 2 k_{2 \min } \\
\sigma_{0} r_{0}, \sigma_{1} r_{1}, \sigma_{2} r_{2}
\end{array}\right\}, \\
c & =\sum_{i=0}^{2}\left[\frac{1}{2} \sigma_{i}\left(\rho_{i}-\rho_{i}^{0}\right)^{2}+\frac{\rho_{i}}{4 \varepsilon_{i}}\right], \\
k_{i \min } & =\min \left\{k_{i 0}, k_{i 1}, k_{i 2}\right\} .
\end{aligned}
$$

Hence, we have

$$
V(t) \leq V(0) e^{-k t}+\frac{c}{k} \leq c_{0}, \quad \forall t \geq 0,
$$

where

$$
c_{0}=V(0)+\frac{c}{k} .
$$

Consequently, the following conclusions can be obtained.
(1) For arbitrary $\sigma_{i}>0$, by choosing proper gain parameters $\mathbf{k}_{i}, r_{i}$, and $\varepsilon_{i}$, the state tracking errors (e.g., $\mathbf{z}_{0}, \mathbf{z}_{1}$, and $\mathbf{z}_{2}$ ) and the unknown parameter tracking errors (e.g., $\widetilde{\rho}_{0}, \widetilde{\rho}_{1}$, and $\widetilde{\rho}_{2}$ ) can converge to the neighborhoods of the origin exponentially; that is,

$$
\boldsymbol{\Omega}=\left\{\mathbf{z}_{0}, \mathbf{z}_{1}, \mathbf{z}_{2}, \tilde{\rho}_{0}, \widetilde{\rho}_{1}, \tilde{\rho}_{2} \mid V \leq \frac{c}{k}\right\} .
$$

The rate and domain of convergence can be regulated by adjusting the values of $\mathbf{k}_{i}, \sigma_{i}, r_{i}$, and $\varepsilon_{i}$. What is more, the algorithm is designed with no information of the upper bound of the uncertainties, which is easier to realize.

\section{Simulation and Analysis}

4.1. Simulation Conditions. To verify the performance of proposed integrated guidance and control algorithm, numerical simulation is conducted for three-dimensional near space interception.

The initial position and velocity vectors of the interceptor with respect to inertial coordinate system are set as

$$
\begin{aligned}
& r_{m}(0)=\left[\begin{array}{lll}
2000 & 4000 & 0
\end{array}\right]^{T} \mathrm{~m}, \\
& v_{m}(0)=\left[\begin{array}{lll}
398.24 & 34.86 & -13.91
\end{array}\right]^{T} \mathrm{~m} / \mathrm{s} .
\end{aligned}
$$

Meanwhile

$$
\begin{gathered}
\alpha(0)=0^{\circ}, \\
\beta(0)=-1^{\circ}, \\
\gamma(0)=2^{\circ}, \\
\omega_{x}(0)=0, \\
\omega_{y}(0)=0, \\
\omega_{z}(0)=0 .
\end{gathered}
$$

The initial position and velocity vectors of the target with respect to inertial coordinate system are set as

$$
\begin{aligned}
& r_{t}(0)=\left[\begin{array}{lll}
5000 & 8000 & 4000
\end{array}\right]^{T} \mathrm{~m}, \\
& v_{t}(0)=\left[\begin{array}{lll}
240 & 0 & 0
\end{array}\right]^{T} \mathrm{~m} / \mathrm{s} .
\end{aligned}
$$

For the purpose of comparison, three guidance and control schemes are carried out in the simulation, details of which are as follows.

(1) The proposed integrated guidance and control algorithm is denoted by IGC. The gain parameters of the IGC algorithm are set as

$$
\begin{aligned}
& \mathbf{k}_{0}=\operatorname{diag}\left(\left[\begin{array}{ll}
0.3 & 0.3
\end{array}\right]\right), \\
& \varepsilon_{0}=0.05, \\
& r_{0}=1, \\
& \sigma_{0}=0.2, \\
& \rho_{0}^{0}=0.01,
\end{aligned}
$$




$$
\begin{aligned}
& \delta_{0}=0.5\left\|\mathbf{f}_{0}\left(\mathbf{x}_{0}\right)\right\|, \\
& \mathbf{k}_{1}=\operatorname{diag}\left(\left[\begin{array}{lll}
1 & 2 & 2
\end{array}\right]\right), \\
& \varepsilon_{1}=0.1, \\
& r_{1}=1, \\
& \sigma_{1}=0.5, \\
& \rho_{1}^{0}=0.01, \\
& \delta_{1}=0.5\left\|\mathbf{f}_{1}\left(\mathbf{x}_{1}\right)\right\|, \\
& \mathbf{k}_{2}=\operatorname{diag}\left(\left[\begin{array}{ll}
2 & 3
\end{array}\right]\right), \\
& \varepsilon_{2}=0.1, \\
& r_{2}=1, \\
& \sigma_{2}=0.5, \\
& \rho_{2}^{0}=0.01, \\
& \delta_{2}=0.5\left\|\mathbf{f}_{2}\left(\mathbf{x}_{1}, \mathbf{x}_{2}\right)\right\| .
\end{aligned}
$$

(2) The proportional navigation guidance law combined with PID control law is denoted by PN+PID. Using PN law, we can get the commanding normal overload with respect to half velocity coordinate system; that is [30],

$$
\begin{aligned}
& n_{y c}=K|\dot{R}| \dot{\varepsilon}, \\
& n_{z c}=K|\dot{R}| \dot{\eta},
\end{aligned}
$$

where the gain parameter is set as $K=6$.

The PID law is used to track the commanding normal overload, which is given by the following [30]:

$$
\begin{aligned}
& \delta_{x}=K_{p x}\left(\gamma_{c}-\gamma\right)+K_{d x}\left(-\omega_{x}\right), \\
& \delta_{y}=K_{i y} \int_{0}^{t}\left(n_{y c}-n_{y}\right) d \tau+K_{d y}\left(-\omega_{z}\right), \\
& \delta_{z}=K_{i z} \int_{0}^{t}\left(n_{z c}-n_{z}\right) d \tau+K_{d z}\left(-\omega_{y}\right),
\end{aligned}
$$

where $K_{p x}=0.0135, K_{d x}=-0.0014, K_{i y}=0.0110, K_{d y}=$ $0.1981, K_{i z}=-0.1260$, and $K_{d z}=0.1949$.

(3) The sliding mode guidance law combined with active disturbance rejection control law is denoted by SMG+ADRC. Using SMG law, we can get the commanding normal acceleration with respect to line-of-sight coordinate system [31]:

$$
\begin{aligned}
a_{M \varepsilon c} & \\
= & -N \dot{R} \dot{\varepsilon}-R \dot{\eta}^{2} \sin \varepsilon \cos \varepsilon \\
& +\left[\left(M_{0}+k_{1} \int_{0}^{t}|R \dot{\varepsilon}| d \tau\right)-K^{\prime} \rho \dot{R}\right] \operatorname{sgn}(\dot{\varepsilon}),
\end{aligned}
$$

$$
\begin{aligned}
& a_{M \eta c} \\
& =N \dot{R} \dot{\eta} \cos \varepsilon-2 R \dot{\varepsilon} \dot{\eta} \sin \varepsilon \\
& \quad-\left[\left(M_{0}+k_{1} \int_{0}^{t}|R \dot{\eta}| d \tau\right)-K^{\prime} \rho \dot{R}\right] \operatorname{sgn}(\dot{\eta}), \\
& \operatorname{sat}(x, \delta)= \begin{cases}1, & (x>\delta), \\
\frac{x}{\delta}, & (|x| \leq \delta), \\
-1, & (x<-\delta),\end{cases}
\end{aligned}
$$

where the gain parameters are set as $N=4, K^{\prime}=2, \rho=0.02$, $M_{0}=10, k_{1}=1.0$, and $\delta=0.0015$. Furthermore, we can get the commanding angle of attack and sideslip, that is, $\alpha_{c}$ and $\beta_{c}$.

The ADRC law is used to track the guidance command, which is given by the following [29]:

$$
\begin{aligned}
& x_{1 c}=\left[\begin{array}{lll}
\alpha_{c} & \beta_{c} & 0
\end{array}\right]^{T}, \\
& \mathbf{e}_{10}=\mathbf{z}_{11}-\mathbf{x}_{1}, \\
& \dot{\mathbf{z}}_{11}=\mathbf{z}_{12}-\beta_{11} \mathbf{e}_{10}+\mathbf{g}_{1} \mathbf{x}_{2}, \\
& \dot{\mathbf{z}}_{12}=-\beta_{12} \mathrm{fal}\left(\mathbf{e}_{10}, \alpha_{10}, \delta_{10}\right), \\
& \mathbf{x}_{2 c}=\mathbf{g}_{1}^{-1}\left[-\mathbf{z}_{12}+\beta_{1} \mathrm{fal}\left(\mathbf{x}_{1 c}-\mathbf{z}_{11}, \alpha_{1}, \delta_{1}\right)\right], \\
& \mathbf{e}_{20}=\mathbf{z}_{21}-\mathbf{x}_{2}, \\
& \dot{\mathbf{z}}_{21}=\mathbf{z}_{22}-\beta_{21} \mathbf{e}_{20}+\mathbf{g}_{2} \mathbf{u}, \\
& \dot{\mathbf{z}}_{22}=-\beta_{22} \mathrm{fal}\left(\mathbf{e}_{20}, \alpha_{20}, \delta_{20}\right), \\
& \mathbf{u}=\mathbf{g}_{2}^{-1}\left[-\mathbf{z}_{22}+\beta_{2} \mathrm{fal}\left(\mathbf{x}_{2 c}-\mathbf{z}_{21}, \alpha_{2}, \delta_{2}\right)\right],
\end{aligned}
$$

where $\beta_{21}=200, \beta_{22}=1, \alpha_{20}=0.5, \delta_{20}=0.01 ; \beta_{2}=50, \alpha_{2}=$ $0.5, \delta_{2}=0.01 ; \beta_{11}=100, \beta_{12}=0.1, \alpha_{10}=0.5, \delta_{20}=0.001$; $\beta_{1}=4, \alpha_{1}=0.5$, and $\delta_{1}=0.001$.

4.2. Results and Analysis. Under conditions above, numerical simulations are carried out in two typical engagement scenarios. For better illustration of proposed methods performance when there are aerodynamic uncertainties, 100 Monte-Carlo runs are performed in each engagement scenario, in which the random variables are the deviation of the aerodynamic parameters from their nominal design values, uniformly distributed in the interval $[-20 \%,+20 \%]$.

Scenario 1 (nonmaneuvering target). Suppose that the target does not maneuver; the mean miss distance and interception time of the three schemes resulting from Monte-Carlo runs are summarized in Table 1. Simulation results of the three schemes are presented in Figures 3-6. (To minimize the article length, only one representative result from Monte-Carlo runs is presented, in which the aerodynamic parameters deviate from their nominal design values by $+20 \%$.)

Scenario 2 (step maneuvering target). Assume that the target escapes with accelerationof $a_{t y}=-2 g$ and $a_{t z}=4 g$. 
TABLE 1: Mean miss distance and interception time of the three schemes in Scenario 1.

\begin{tabular}{lcc}
\hline Scheme & Mean miss distance & Mean interception time \\
\hline IGC & $0.63 \mathrm{~m}$ & $37.1 \mathrm{~s}$ \\
PN+PID & $1.21 \mathrm{~m}$ & $38.2 \mathrm{~s}$ \\
SMG+ADRC & $0.95 \mathrm{~m}$ & $37.7 \mathrm{~s}$
\end{tabular}

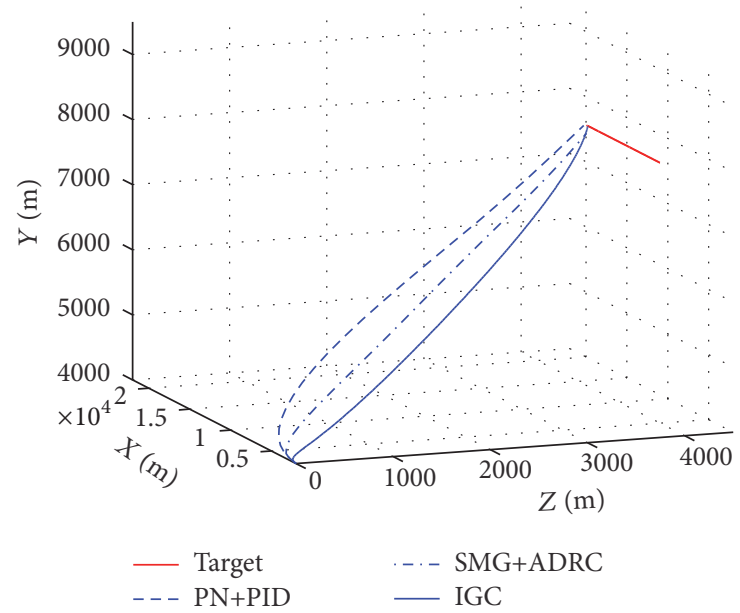

FIgURE 3: The interceptor-target relative motion in Scenario 1.

TABLE 2: Mean miss distance and interception time of the three schemes in Scenario 2.

\begin{tabular}{lcc}
\hline Scheme & Mean miss distance & Mean interception time \\
\hline IGC & $0.71 \mathrm{~m}$ & $22.2 \mathrm{~s}$ \\
PN+PID & $2.32 \mathrm{~m}$ & $23.2 \mathrm{~s}$ \\
SMG+ADRC & $1.45 \mathrm{~m}$ & $22.7 \mathrm{~s}$ \\
\hline
\end{tabular}

The mean miss distance and interception time of the three schemes resulting from Monte-Carlo runs are summarized in Table 2. Simulation results of the three schemes are presented in Figures 7-10. (To minimize the article length, only one representative result from Monte-Carlo runs is presented, in which the aerodynamic parameters deviate from their nominal design values by $+20 \%$.)

Simulation results above indicate that, comparing to conventional design methods, the integrated guidance and control algorithm is more effective with the following advantages:

(1) less miss distance and interception time, which is not obvious when attacking nonmaneuvering target while it is rather remarkable when attacking maneuvering target;

(2) smaller attack angle and sideslip angle, hence reduced required normal overload and less fluctuation of trajectory; therefore, the integrated design excels in maneuvering target interception especially when the available normal overload is limited;
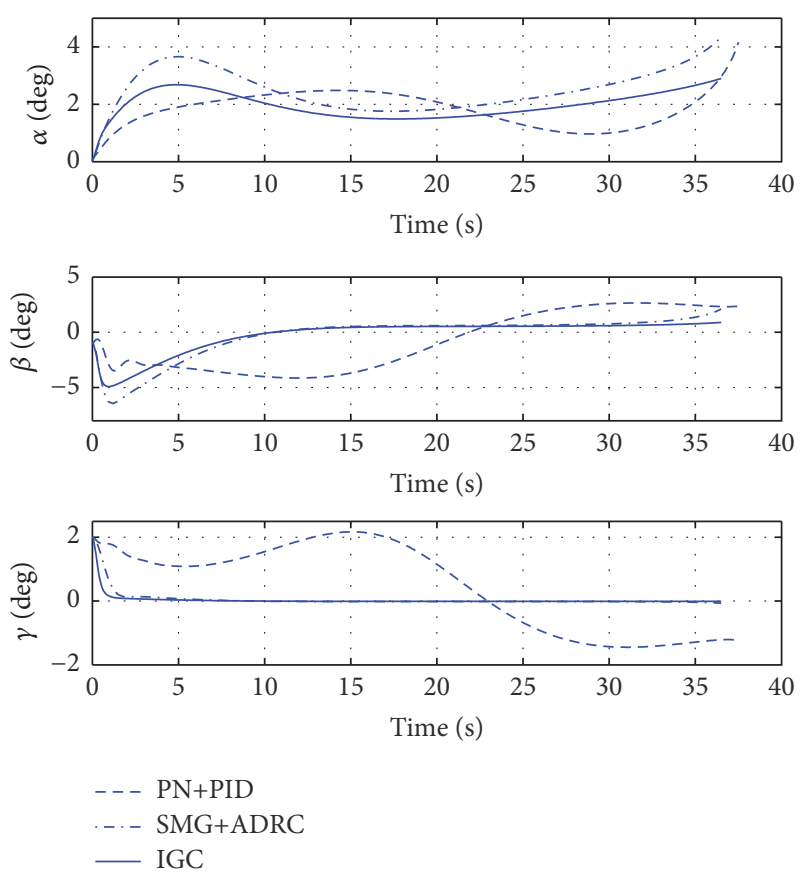

FIGURE 4: The range of attack angle, sideslip angle, and roll angle with respect to time in Scenario 1.
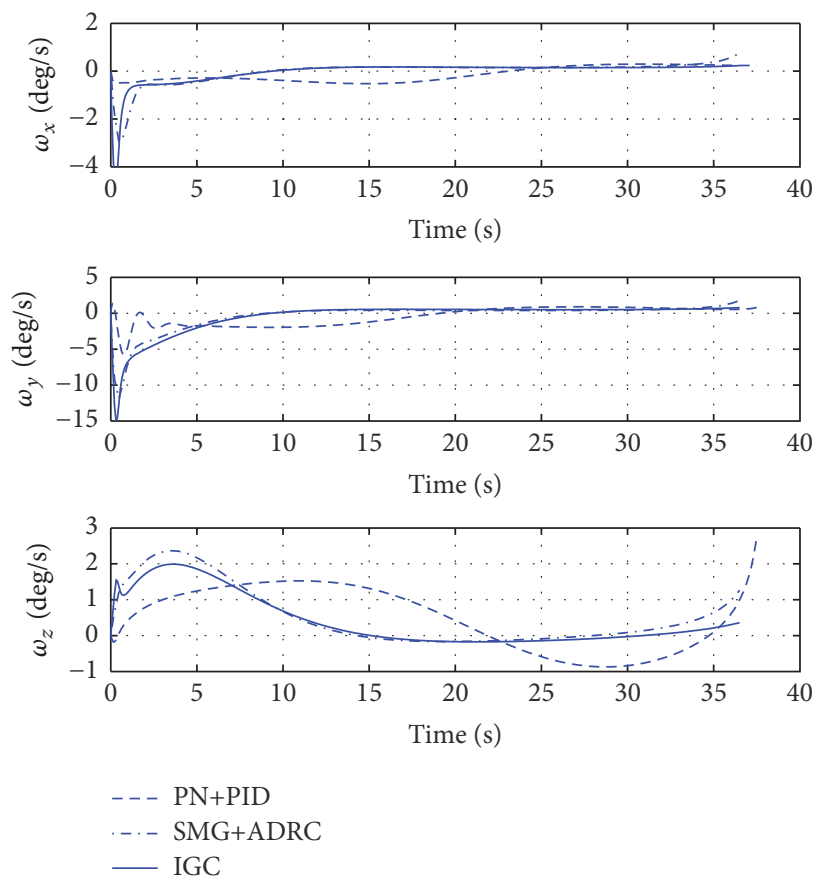

Figure 5: The range of rotational angular velocity with respect to time in Scenario 1.

(3) higher flight stability, with the attack angle, sideslip angle, roll angle, rotational angular velocity, and deflection of aerodynamic fins remaining stable during the whole flight, while those of conventional design schemes tend to diverge when the interceptor approaches the target. 

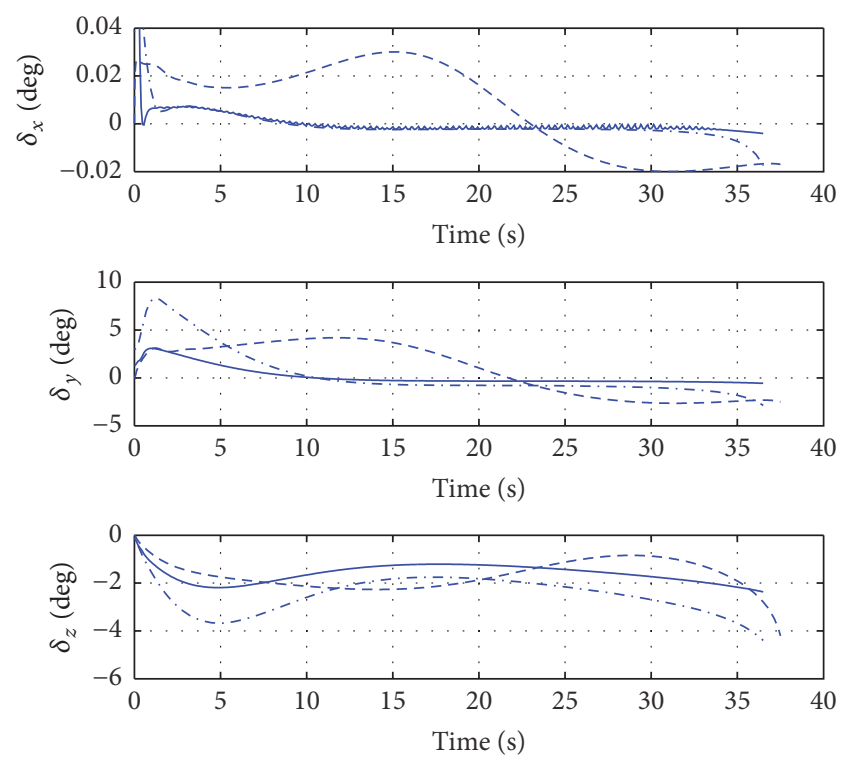

- - - PN+PID

-. SMG+ADRC

- IGC

FIGURE 6: The range of deflections of aerodynamic fins with respect to time in Scenario 1.

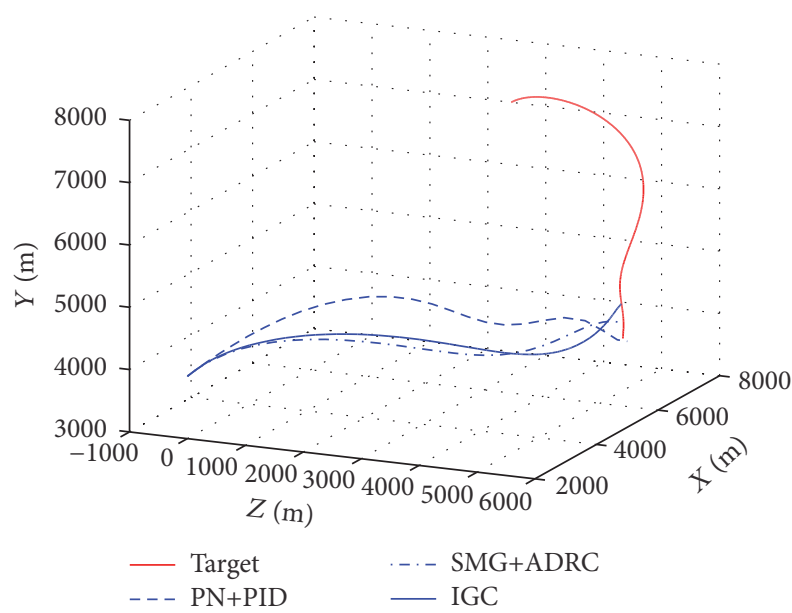

FIGURE 7: The interceptor-target relative motion in Scenario 2.

\section{Conclusion}

Aiming at three-dimensional near space interception of maneuvering target, a novel integrated guidance and control method considering the coupling among three channels is proposed in this paper. Firstly, considering the coupling among the pitch, yaw, and roll channels, three-dimensional integrated guidance and control model is employed by combining the interceptor-target relative motion model with the nonlinear dynamics of the interceptor, in which the direct relationship between the interceptor-target relative motion and the deflections of aerodynamic fins is established. Secondly, regarding the acceleration of the target as bounded uncertainty of the system, an integrated guidance and control
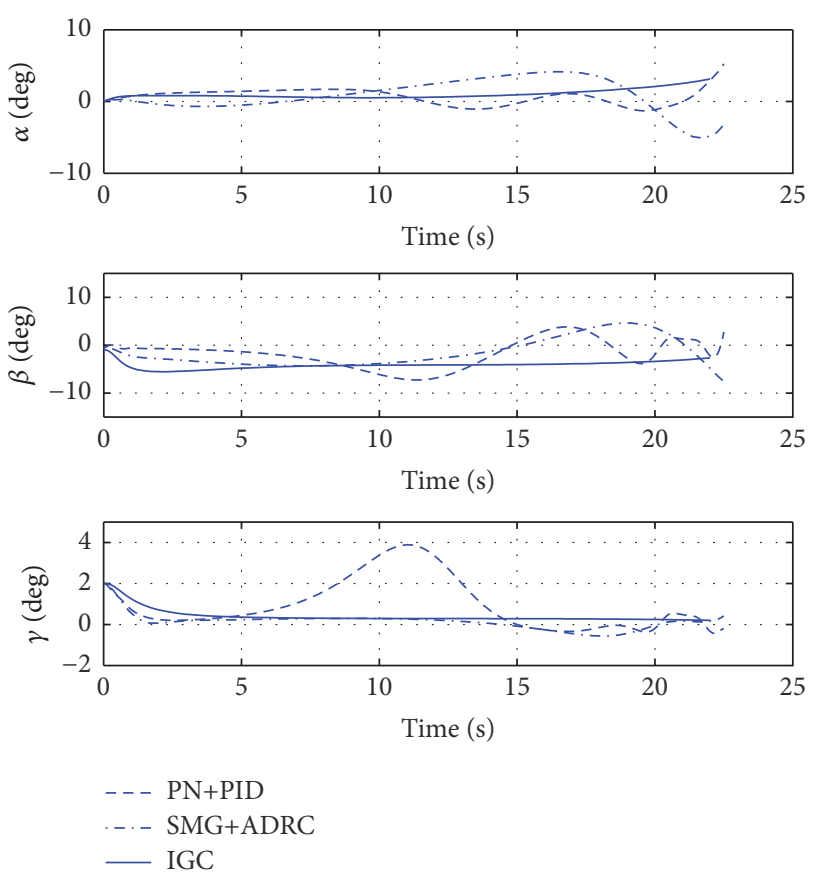

FIGURE 8: The range of attack angle, sideslip angle, and roll angle with respect to time in Scenario 2.
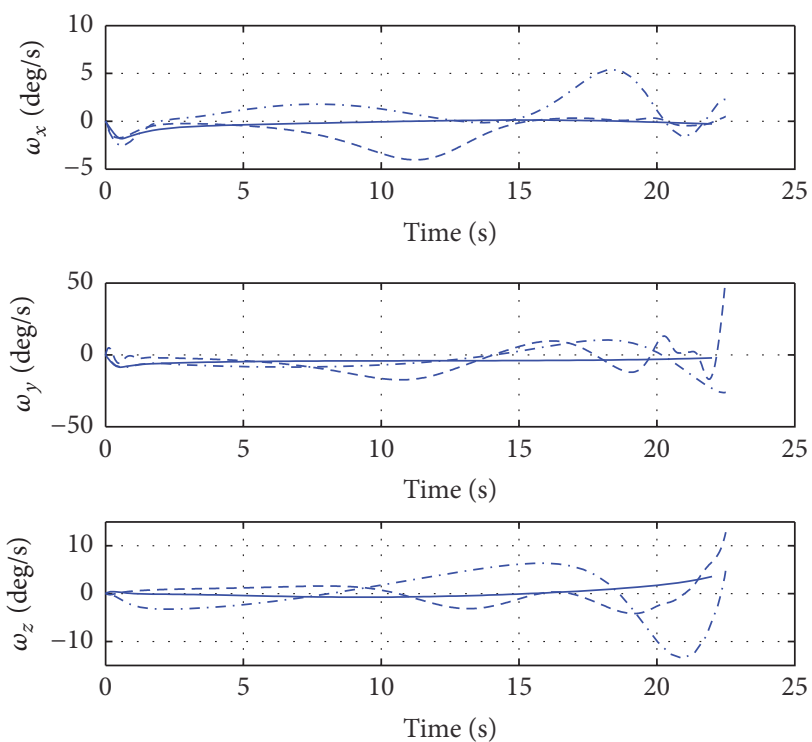

$$
\begin{array}{ll}
--- & \text { PN+PID } \\
\text {-.- } \text { SMG+ADRC } \\
\text { - IGC }
\end{array}
$$

FIGURE 9: The range of rotational angular velocity with respect to time in Scenario 2.

algorithm is designed based on robust adaptive backstepping method, with the upper bound of the uncertainties unknown. Moreover, a nonlinear tracking differentiator is introduced to reduce the "compute explosion" caused by backstepping method. It is proved that tracking errors of the state and 

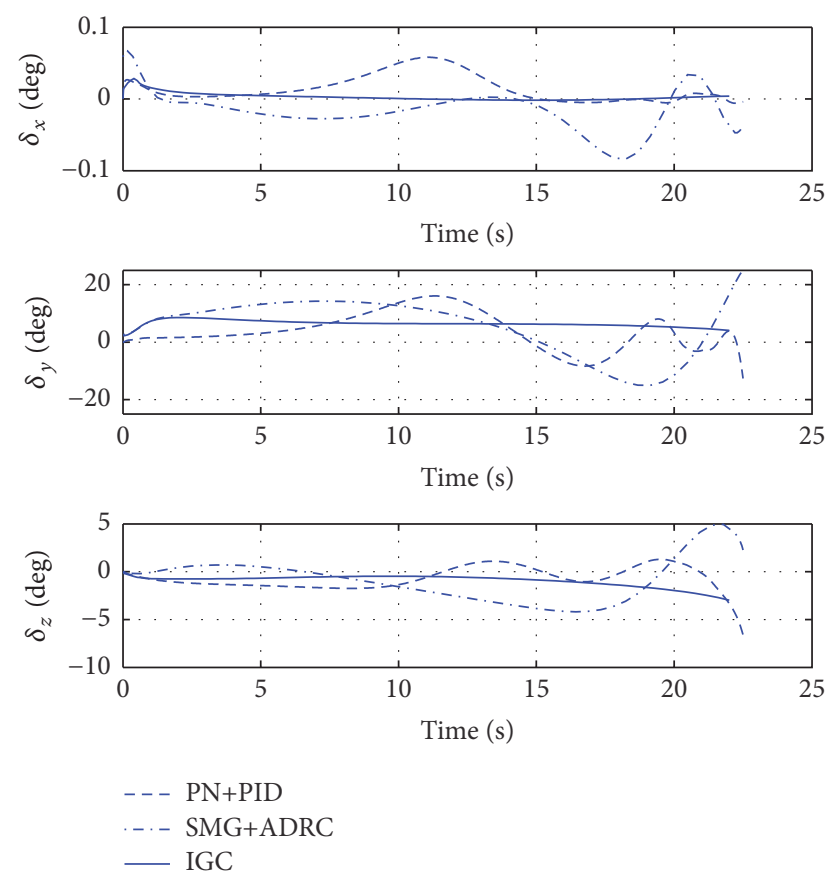

FIGURE 10: The range of deflections of aerodynamic fins with respect to time in Scenario 2.

the upper bound of the uncertainties converge to the neighborhoods of the origin exponentially. Finally, simulation and comparison are carried out under three guidance and control schemes. In contrast to conventional design methods, the integrated guidance and control algorithm leads to higher flight stability, less miss distance, and required normal overload, especially when attacking the high-maneuvering target.

\section{Nomenclature}

\begin{tabular}{|c|c|}
\hline$R:$ & Line-of-sight distance $(\mathrm{m})$ \\
\hline$V:$ & Velocity of interceptor (m/s) \\
\hline$\varepsilon:$ & Elevation angle of the line-of-sight (rad) \\
\hline$\eta:$ & Azimuth angle of the line-of-sight (rad) \\
\hline$\theta$ & Flight path angle (rad) \\
\hline$\psi_{v}:$ & Pitch angle (rad) \\
\hline$\alpha:$ & Angle of attack (rad) \\
\hline$\beta:$ & Angle of sideslip (rad) \\
\hline$\gamma:$ & Roll angle (rad) \\
\hline$\omega_{x}, \omega_{y}, \omega_{z}:$ & Roll, yaw, and pitch rates ( $\mathrm{rad} / \mathrm{s})$ \\
\hline$\delta_{x}, \delta_{y}, \delta_{z}:$ & $\begin{array}{l}\text { Aileron, rudder, and elevator deflections } \\
\text { (rad) }\end{array}$ \\
\hline$m:$ & Mass of interceptor (kg) \\
\hline$q:$ & Dynamic pressure $(\mathrm{Pa})$ \\
\hline S: & Reference area $\left(\mathrm{m}^{2}\right)$ \\
\hline$L:$ & Reference length (m) \\
\hline$I_{x}, I_{y}, I_{z}:$ & $\begin{array}{l}\text { Roll, yaw, and pitch moments of inertia } \\
\left(\mathrm{kg} \cdot \mathrm{m}^{2}\right)\end{array}$ \\
\hline$C_{y}^{\alpha}:$ & $\begin{array}{l}\text { Partial derivative of lift force coefficient } \\
\text { with respect to } \alpha\end{array}$ \\
\hline$C_{z}^{\beta}$ & $\begin{array}{l}\text { Partial derivative of lateral force coefficient } \\
\text { with respect to } \beta\end{array}$ \\
\hline
\end{tabular}

$m_{x}^{\bar{\omega}_{x}}, m_{x}^{\delta_{x}}: \quad$ Partial derivatives of rolling moment coefficient with respect to $\omega_{x}$ and $\delta_{x}$

$m_{y}^{\beta}, m_{y}^{\bar{\omega}_{y}}, m_{y}^{\delta_{y}}$ : Partial derivatives of yawing moment coefficient with respect to $\beta, \omega_{y}$ and $\delta_{y}$

$m_{z}^{\alpha}, m_{z}^{\bar{\omega}_{z}}, m_{z}^{\delta_{z}}$ : Partial derivatives of pitching moment coefficient with respect to $\alpha, \omega_{z}$ and $\delta_{z}$.

\section{Competing Interests}

The authors declare that there are no competing interests regarding the publication of this paper.

\section{Acknowledgments}

This work was partially supported by the National Natural Science Foundation of China (Grant no. 11572097).

\section{References}

[1] R. H. Chen, J. L. Speyer, and D. Lianos, "Optimal intercept missile guidance strategies with autopilot lag," Journal of Guidance, Control, and Dynamics, vol. 33, no. 4, pp. 1264-1272, 2010.

[2] S. S. Moosapour, G. Alizadeh, S. Khanmohammadi, and H. Moosapour, "A novel robust proportional navigation guidance law design for missile considering autopilot dynamic," Transactions of the Institute of Measurement and Control, vol. 35, no. 5, pp. 703-710, 2013.

[3] W. Shang, J. Guo, S. Tang, Y. Ma, and Y. Zhang, "Impact time guidance law considering autopilot dynamics based on variable coefficients strategy for maneuvering target," Mathematical Problems in Engineering, vol. 2015, Article ID 815149, 10 pages, 2015.

[4] D. Chwa and J. Y. Choi, "Adaptive nonlinear guidance law considering control loop dynamics," IEEE Transactions on Aerospace and Electronic Systems, vol. 39, no. 4, pp. 1134-1143, 2003.

[5] B. Xu and D. Zhou, "Three dimensional adaptive dynamic surface guidance law accounting for autopilot lag," in Proceedings of the American Control Conference (ACC '14), pp. 578-583, IEEE, Portland, Ore, USA, June 2014.

[6] P. P. Qu and D. Zhou, "A dimension reduction observer-based guidance law accounting for dynamics of missile autopilot," Proceedings of the Institution of Mechanical Engineers, Part G: Journal of Aerospace Engineering, vol. 227, no. 7, pp. 1114-1121, 2013.

[7] G.-L. Li, H. Yan, and H.-B. Ji, "A guidance law with finite time convergence considering autopilot dynamics and uncertainties," International Journal of Control, Automation and Systems, vol. 12, no. 5, pp. 1011-1017, 2014.

[8] S. M. He and D. F. Lin, "Continuous composite finite-time convergent guidance laws with autopilot dynamics compensation," ISA Transactions, vol. 58, pp. 270-278, 2015.

[9] P. Qu, C. Shao, and D. Zhou, "Finite time convergence guidance law accounting for missile autopilot," Journal of Dynamic Systems, Measurement and Control, Transactions of the ASME, vol. 137, no. 5, Article ID 051014, pp. 1-8, 2015.

[10] Z. X. Zhang, S. H. Li, and S. Luo, "Composite guidance laws based on sliding mode control with impact angle constraint and autopilot lag," Transactions of the Institute of Measurement and Control, vol. 35, no. 6, pp. 764-776, 2013. 
[11] S. He, D. Lin, and J. Wang, "Robust terminal angle constraint guidance law with autopilot lag for intercepting maneuvering targets," Nonlinear Dynamics, vol. 81, no. 1-2, pp. 881-892, 2015.

[12] D. Zhou, P. P. Qu, and S. Sun, "A guidance law with terminal impact angle constraint accounting for missile autopilot," Journal of Dynamic Systems, Measurement and Control, vol. 135, no. 5, Article ID 051009, pp. 1-10, 2013.

[13] H. Yan, X. Wang, B. Yu, and H. Ji, "Adaptive integrated guidance and control based on backstepping and input-to-state stability," Asian Journal of Control, vol. 16, no. 2, pp. 602-608, 2014.

[14] M. F. Jegarkandi, A. Ashrafifar, and R. Mohsenipour, "Adaptive integrated guidance and fault tolerant control using backstepping and sliding mode," International Journal of Aerospace Engineering, vol. 2015, Article ID 253478, 7 pages, 2015.

[15] S. Xingling and W. Honglun, "Back-stepping active disturbance rejection control design for integrated missile guidance and control system via reduced-order ESO," ISA Transactions, vol. 57, pp. 10-22, 2015.

[16] A. Zhurbal and M. Idan, "Effect of estimation on the performance of an integrated missile guidance and control system," IEEE Transactions on Aerospace and Electronic Systems, vol. 47, no. 4, pp. 2690-2708, 2011.

[17] H. Yan and H. Ji, "Integrated guidance and control for dualcontrol missiles based on small-gain theorem," Automatica, vol. 48, no. 10, pp. 2686-2692, 2012.

[18] K. Chen, B. Fu, Y. Ding, and J. Yan, "Integrated guidance and control method for the interception of maneuvering hypersonic vehicle based on high order sliding mode approach," Mathematical Problems in Engineering, vol. 2015, Article ID 648231, 19 pages, 2015.

[19] S. Shamaghdari, S. K. Nikravesh, and M. Haeri, "Integrated guidance and control of elastic flight vehicle based on robust MPC,' International Journal of Robust and Nonlinear Control, vol. 25, no. 15, pp. 2608-2630, 2015.

[20] M.-Z. Hou and G.-R. Duan, "Adaptive dynamic surface control for integrated missile guidance and autopilot," International Journal of Automation and Computing, vol. 8, no. 1, pp. 122-127, 2011.

[21] X. L. Liang, M. Z. Hou, and G. R. Duan, "Adaptive dynamic surface control for integrated missile guidance and autopilot in the presence of input saturation," Journal of Aerospace Engineering, vol. 28, no. 5, Article ID 04014121, pp. 1-8, 2015.

[22] F. Wang, G. Liu, and X.-G. Liang, "Integrated guidance and control design for the near space interceptor," International Journal of Aeronautical and Space Sciences, vol. 16, no. 2, pp. 278-294, 2015.

[23] H. T. Song, T. Zhang, G. L. Zhang, and C. J. Lu, "Integrated interceptor guidance and control with prescribed performance," International Journal of Robust and Nonlinear Control, vol. 25, no. 16, pp. 3179-3194, 2015.

[24] M. Z. Hou, X. L. Liang, and G. R. Duan, "Adaptive block dynamic surface control for integrated missile guidance and autopilot," Chinese Journal of Aeronautics, vol. 26, no. 3, pp. 741750, 2013.

[25] H. Song and T. Zhang, "Fast robust integrated guidance and control design of interceptors," IEEE Transactions on Control Systems Technology, vol. 24, no. 1, pp. 349-356, 2016.

[26] C. Guo and X.-G. Liang, "Integrated guidance and control based on block backstepping sliding mode and dynamic control allocation," Proceedings of the Institution of Mechanical Engineers Part G: Journal of Aerospace Engineering, vol. 229, no. 9, pp. 1559-1574, 2015.
[27] H. T. Song, T. Zhang, and G. L. Zhang, " $\mathrm{L}_{1}$ adaptive state feedback controller for three-dimensional integrated guidance and control of interceptor," Proceedings of the Institution of Mechanical Engineers Part G: Journal of Aerospace Engineering, vol. 228, no. 10, pp. 1693-1701, 2014.

[28] Y. A. Hu, Y. Q. Jin, and H. Y. Li, Robustness of Adaptive Backstepping Control for Nonlinear Systems, Publishing House of Electronics Industry, Beijing, China, 2010.

[29] J. Q. Han, Active Disturbance Rejection Control Technique-The Technique for Estimating and Compensating the Uncertainties, National Defense Industry Press, 2009.

[30] X. F. Qian, R. X. Lin, and Y. N. Zhao, Flight Dynamics of Missile, Beijing Institute of Technology Press, Beijing, China, 2008.

[31] D. Zhou, New Guidance Laws for Homing Missile, National Defense Industry Press, 2002. 


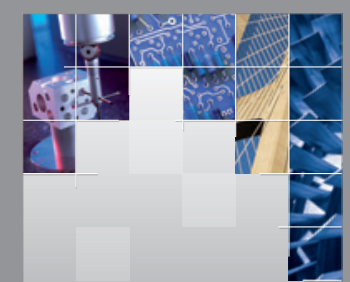

\section{Enfincering}
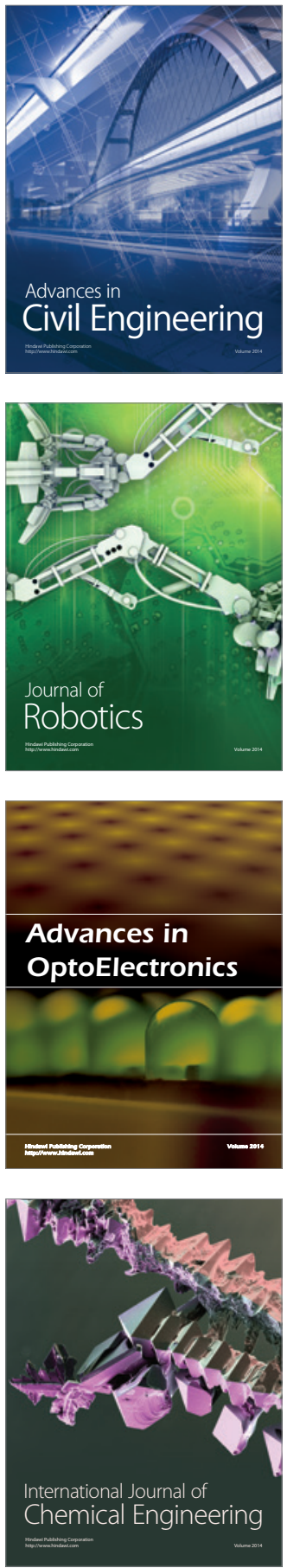

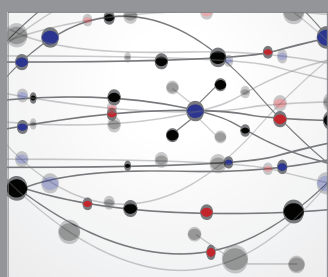

The Scientific World Journal

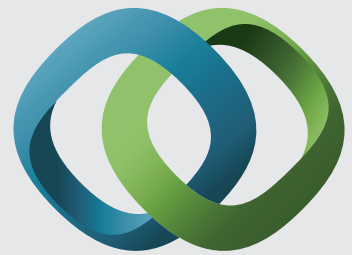

\section{Hindawi}

Submit your manuscripts at

http://www.hindawi.com
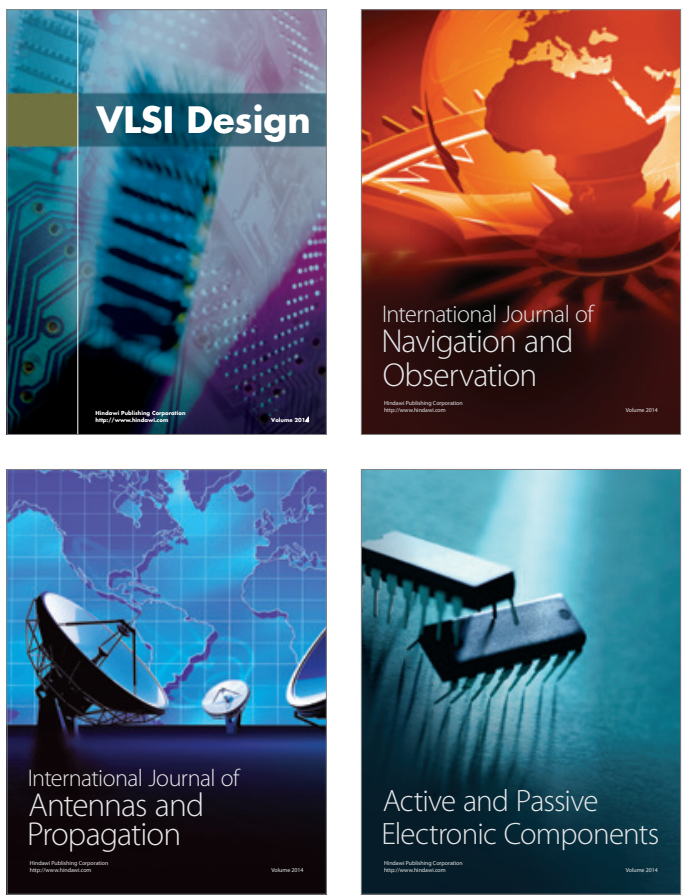
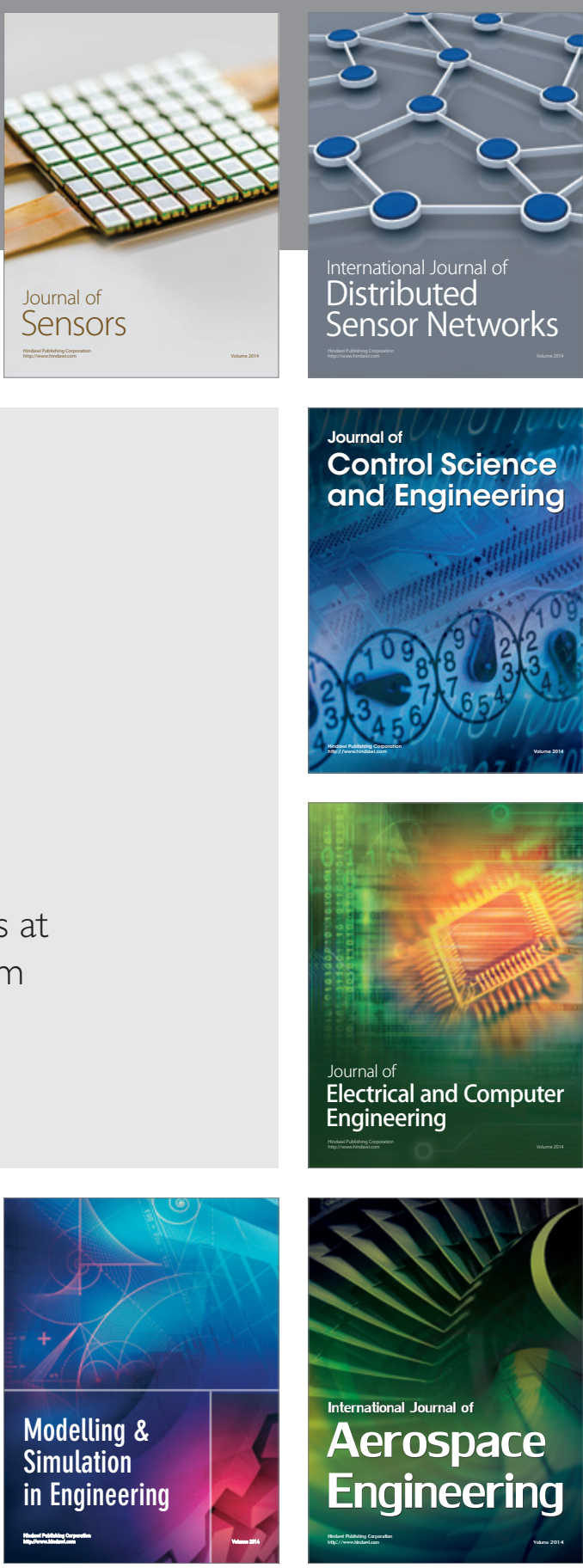

International Journal of

Distributed

Sensor Networks

Journal of

Control Science

and Engineering
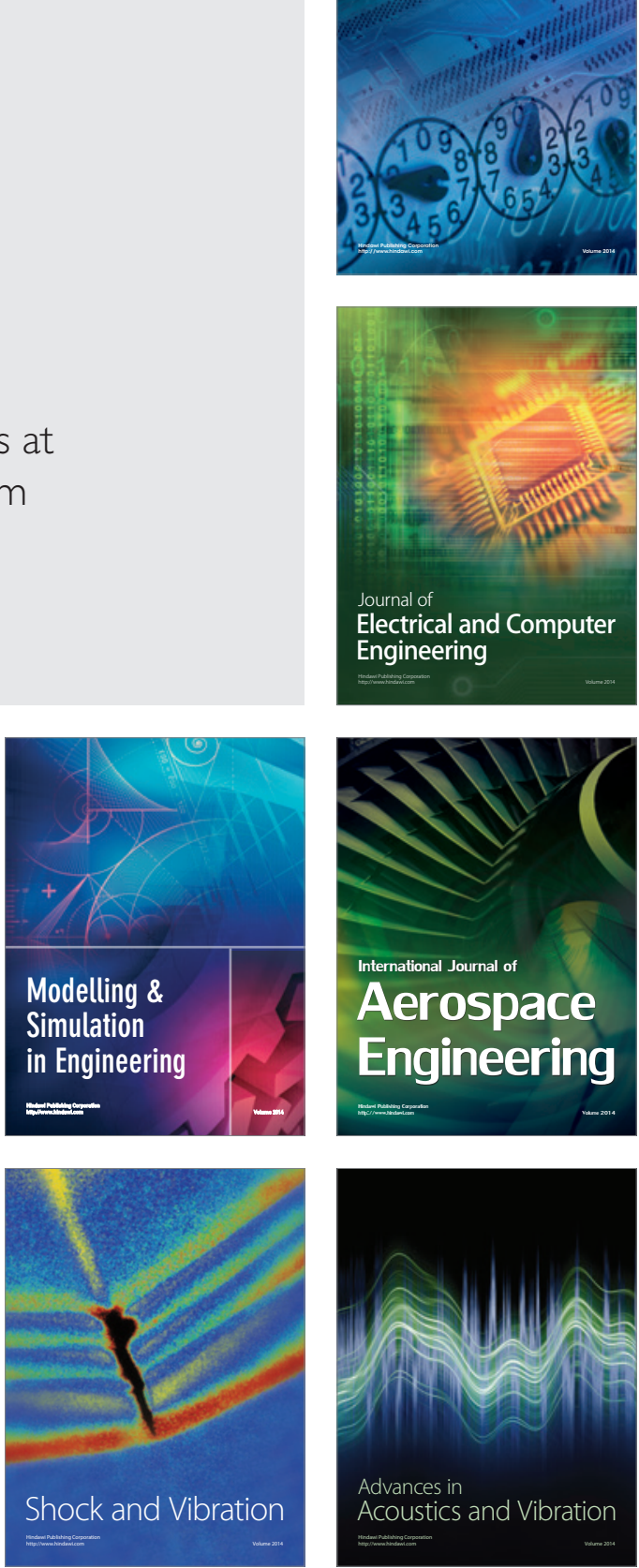